\title{
Auditory Evoked Potential in Patients with Tinnitus
}

\author{
Yi Chen, BS ${ }^{1}$; Chin-Lung Kuo, MD, PhD ${ }^{1,2^{*}}$
}

1 Department of Otolaryngology-Head and Neck Surgery, Taoyuan Armed Forces General Hospital, Taoyuan, Taiwan ${ }^{2}$ Institute of Brain Science, National Yang-Ming University School of Medicine, Taipei, Taiwan

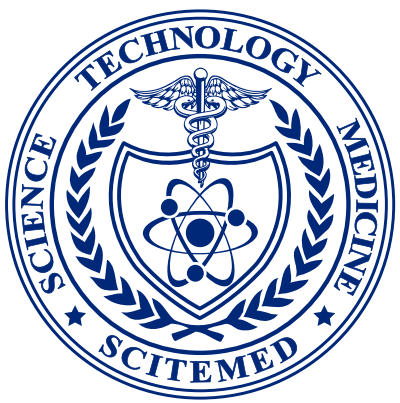

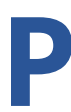
erceiving sounds in the absence of stimulus from an external sound source is referred to as tinnitus [1-3]. Unlike auditory hallucinations, tinnitus is generally an indication of damage to the auditory system, which may be accompanied by hearing loss and vertigo. Tinnitus may result from acoustic trauma and has even been reported among patients with depression. Research has shown that tinnitus occurs in approximately $10-25 \%$ of the world's population; however, only $0.5 \%-1.6 \%$ seeks medical assistance $[1,4]$. In some cases, the symptoms may be severe enough to affect sleep and everyday activities. Patients with tinnitus may be prone emotional exhaustion, anxiety, and poor concentration.

Opinions differ as to the causes of tinnitus. Until 1980, it was believed that tinnitus originated in the hair cells of the cochlea [3]. Even today, the physiological mechanisms underlying tinnitus are still not completely understood. Several scholars have advocated that tinnitus stems from cochlear lesions; however, opinions differ regarding whether tinnitus is caused by the activation or suppression of auditory nerves in the cochlea. Evans et al. claimed that auditory nerves in the cochlea of tinnitus patients are suppressed [5]. However, recent research has revealed that the peripheral auditory system is not the only source of tinnitus [6]. In fact, the pathways from the cochlea to the auditory cortex have been implicated in this condition. There is even the possibility that psychological factors play a role, and researchers have noted a correlation between tinnitus and exposure to high-stress environments for prolonged periods of time [1]. Studies have shown a correlation between tinnitus and abnormal cognitive function [2]. This would indicate that the causes of tinnitus extend beyond the auditory system to higher-order cognitive processing.

Numerous researchers are looking into event-related potentials and their link to tinnitus. It appears that tinnitus patients are more susceptible than normal individuals to cognitive processing issues, in terms of information recognition and the analysis of auditory endogenous sounds. Overall, the causes of tinnitus extends beyond the auditory system to cognitive processing, psychological factors, and even emotional stress [7].

The human auditory system consists of the outer ear, the middle ear, and the inner ear. The outer ear includes the auricle and ear canal. The middle ear cavity houses the ossicular chain through which sounds are amplified and transmitted into the inner ear. The inner ear comprises the cochlea and auditory nerves. Sound waves received by the ear undergo a series of transductions, from sound waves into mechanical energy, which is then converted into electric signals by the cochlea in the form of nerve impulses. No sounds are perceived until these nerve impulses are processed by the brain. Electrical signals from the nervous system can be detected by epidermal and intra-epidermal electrodes. The signals produced by the nervous system in response to specific stimulation are referred to as evoked potentials. In cases where the stimulation source is a sound signal, the responses are called auditory evoked potentials. Based on a specific time frame, these potentials can be further classified as short, middle, or long latency potentials. Short latency potentials can be recorded using the following methods: electrocochleography, auditory steady-state response, and auditory brainstem response. Middle latency potentials include the auditory middle latency response. Long latency potentials include long latency response, P300, and mismatch negativity [8,9].
Tinnitus is not caused merely by the peripheral auditory system. It is possible that the central auditory system also plays a significant role, which means that tinnitus may also be associated with cognitive processing abnormalities or attention deficit disorders. Auditory evoked potentials provide a non-invasive means by which to record the electrical signals of neural activities from the scalp, thus provide a useful tool for the evaluation of auditory disorder, such as tinnitus and hearing loss [10].

\section{ARTICLE INFORMATION}

*Correspondence: Chin-Lung Kuo, MD, PhD, Department of Otolaryngology, Taoyuan Armed Forces General Hospital, No.168, Zhongxing Rd., Longtan Dist, Taoyuan City 32551, Taiwan. E-mail: drkuochinlung@gmail.com

Received:Jan. 10, 2020; Accepted: Feb. 03, 2020; Published: Mar. 31, 2020

\section{DOI: $10.24983 /$ scitemed.aohns.2020.00125}

Ethics Approval and Consent to Participate: The study is in accordance with the ethical standards of the 1964 Helsinki declaration and its later amendments or comparable ethical standards.

Funding: This study was sponsored by grants from Medical Affairs Bureau Ministry of National Defense (MAB-107-099) and Taoyuan Armed Forces General Hospital (AFTYGH No. 10507, AFTYGH No. 10626, and AFTYGH No. 10734).

Conflict of Interest: The authors report no financial or other conflict of interest relevant to this article, which is the intellectual property of the authors.

Copyright $\odot 2020$ The Authors. This is an open-access article distributed under the terms of the Creative Commons Attribution 4.0 International License (CC-BY).

\section{REFERENCES}

1. Konadath $S$, Manjula P. Auditory brainstem response and late latency response in individuals with tinnitus having normal hearing. Intractable Rare Dis Res 2016;5(4):262-268.

2. Gabr TA, El-Hay MA, Badawy A. Electrophysiological and psychological studies in tinnitus. Auris Nasus Larynx 2011;38(6):678-683.

3. Santos Filha VA, Matas CG. Late auditory evoked potentials in individuals with tinnitus. Braz J Otorhinolaryngol 2010;76(2):263-270.

4. Attias J, Urbach D, Gold S, Shemesh Z. Auditory event related potentials in chronic tinnitus patients with noise induced hearing loss. Hear Res 1993;71(1-2):106-113.

5. Evans EF, WilsonJP, Borerwe TA. Animal models of tinnitus. Ciba Found Symp 1981;85:108138.

6. Henry JA, Roberts LE, Caspary DM, Theodoroff SM, Salvi RJ. Underlying mechanisms of tinnitus: review and clinical implications. J Am Acad Audiol 2014;25(1):5-22; quiz 126.

7. Haider HF, Bojic T, Ribeiro SF, Paco J, Hall DA, Szczepek AJ. Pathophysiology of subjective tinnitus: triggers and maintenance. Front Neurosci 2018;12:866.

8. Milloy V, Fournier P, Benoit D, Norena A, Koravand A. Auditory brainstem responses in tinnitus: a review of who, how, and what? Front Aging Neurosci 2017;9:237.

9. Melcher JR, Kiang NYS. Generators of the brainstem auditory evoked potential in cat III: identified cell populations. Hear Res 1996;93(1-2):52-71.

10. Kuo CL. Neuroplastic effect of constraint-induced music therapy on hearing recovery in patients with sudden sensorineural hearing loss. Arch Otorhinolaryngol Head Neck Surg 2019;2(1):3. 